\title{
Effect of high tibial osteotomy on joint loading in symptomatic patients with varus aligned knees: a study using SPECT/CT
}

\author{
Armin Mucha • Milos Dordevic • Anna Hirschmann • \\ Helmut Rasch • Felix Amsler • Markus P. Arnold • \\ Michael T. Hirschmann
}

Received: 13 April 2013/ Accepted: 2 May 2014/Published online: 11 May 2014

(c) Springer-Verlag Berlin Heidelberg 2014

\begin{abstract}
Purpose The purpose was to prospectively evaluate the outcome, in particular the SPECT/CT bone tracer uptake (BTU) after high tibial osteotomy (HTO) due to symptomatic varus malalignment. It was the hypothesis that the BTU after HTO decreases in the medial compartment, clinical outcome and the degree of correction correlates with BTU and asymptomatic patients after HTO reveals a significantly decreased BTU in the medial subchondral areas.

Methods Twenty-two consecutive patients with 23 knees undergoing medial opening-wedge HTO for medial compartment overloading were assessed pre- and postoperatively (12 and/or 24 months) using Tc-99m-HDP-SPECT/ CT including our 4D-SPECT/CT protocol. BTU was quantified and localized to specific biomechanically relevant joint areas. Maximum absolute and relative values (mean \pm standard deviation, median and range) for each area were recorded. Pre- and postoperative mechanical
\end{abstract}

A. Mucha · M. Dordevic · M. P. Arnold .

M. T. Hirschmann ( $\square)$

Department of Orthopaedic Surgery and Traumatology,

Kantonsspital Baselland, 4101 Bruderholz, Switzerland

e-mail: Michael.Hirschmann@ksbl.ch;

Michael.Hirschmann@unibas.ch

\author{
A. Hirschmann \\ Department of Radiology, University Hospital Basel, Basel, \\ Switzerland \\ H. Rasch \\ Institute for Radiology and Nuclear Medicine, Kantonsspital \\ Baselland, 4101 Bruderholz, Switzerland \\ F. Amsler \\ Amsler Consulting, Basel, Switzerland
}

alignment was measured. At 24 months after HTO, the WOMAC score was used.

Results A significant decrease of BTU in the medial subchondral zones after HTO was found (preoperatively to 12 and 24 months postoperatively, $p<0.01$ ). BTU normalized in all asymptomatic patients within 24 months. This decrease was partly seen in the lateral compartments, but significantly higher in the medial compartments $(p<0.0001)$. A significant increase of the BTU was noted in zones directly adjacent to the plate or within the osteotomy zone $(p<0.01)$. Decreased BTU was observed in osteotomy zones at 24 months postoperatively following higher uptake values at 12 months postoperatively. The average valgus correction of the tibiofemoral angle was $5.9^{\circ} \pm 2.8^{\circ}$. Less stiffness correlated significantly with a higher decrease in BTU $(p<0.05)$. Higher postoperative BTU significantly correlated with more pain $(p<0.05)$. No statistical significant associations between BTU and alignment correction were found.

Conclusion In patients with medial compartment, overloading due to varus malalignment HTO led to a significant decrease in BTU in the medial joint compartments. SPECT/CT BTU patterns and intensity in these patients pre- to 12 and 24 months postoperatively were seen. These correlated significantly with pain and stiffness. Hence, SPECT/CT could be used for assessment of adequate correction and healing after HTO. SPECT/CT could be further used to identify the optimal individualized correction for each patient and clinical scenario.

Clinical evidence Diagnostic prospective study, Level II.

Keywords Knee - SPECT/CT · High tibial osteotomy · Localization scheme · Varus malalignment .

Loading history 


\section{Introduction}

Mechanical varus alignment of the knee is a common deformity not always related to clinical symptoms, but known to be related to the development and progression of knee osteoarthritis (OA) [4, 6, 9, 22, 32]. Based on combined investigations of mechanical alignment and gait, Johnson et al. [18] showed that the knee is predominantly loaded in the medial compartment, even in a neutrally aligned leg. In a knee with neutral mechanical alignment, approximately 60-70\% of the loading forces affect the medial tibiofemoral joint. From this fact, it is clear that even a mild varus alignment could result in medial knee pain and progressive medical compartment overloading. To avoid medial compartment degeneration, it needs to be identified at an early stage, and only then progression of OA could be delayed or even prevented surgically. Jackson and Waugh [17] and Coventry et al. [5] were the first to propose high tibial osteotomy (HTO) as a viable, jointpreserving treatment option for medial compartment OA in varus deformed knees.

Current routine radiological imaging before HTO includes anterior-posterior and lateral weight-bearing, long leg radiographs [8] as well as a $45^{\circ}$ posterior-anterior view [28]. These radiographs are able to identify late OA changes such as joint space narrowing, subchondral sclerosis and osteophyte formation, but fail to show overloading or early degenerative changes [37]. Postoperative radiographs are also not capable of revealing the unloading effect after HTO.

Recently, it was shown that combined single photon emission-computerized tomography and conventional computerized tomography (SPECT/CT) is able to visualize mechanical overloading of joint compartments. More specifically, SPECT/CT tracer uptake was correlated with anatomical and mechanical alignment [15]. The intensity of SPECT/CT tracer uptake in the medial and lateral knee compartment significantly correlated with varus or valgus alignment of the knee [15]. A higher degree of radiographically evident osteoarthritis was significantly related to higher tracer uptake in the corresponding joint compartments [15]. It was concluded that SPECT/CT reflects the specific loading pattern of the knee with regard to its alignment [15].

The purpose of this prospective study was to evaluate the clinical and radiological outcome after HTO due to medial compartment overloading. In particular, the SPECT/CT bone tracer uptake (BTU) pattern and intensity were evaluated over the course of time. It was the hypothesis that the BTU after HTO decreases and then normalizes in the medial compartment, clinical outcome and the degree of correction correlates with BTU and asymptomatic patients after HTO reveals a significantly decreased and normalized BTU in the medial subchondral areas.

\section{Materials and methods}

Twenty-two consecutive patients (male/female 18:4, mean age $47 \pm 10$ ) with twenty-three knees (male/female 19:4) undergoing medial opening-wedge HTO for medial joint compartment overloading or medial tibiofemoral osteoarthritis due to mechanical varus alignment were prospectively included in this study. All subjects gave written informed consent before inclusion into the study. Exclusion criteria were posttraumatic osteoarthritis, open growth plates, corticosteroid use within last 6 months, known history of avascular necrosis, osteochondrosis dissecans, chondrocalcinosis, tumour, Paget's disease, joint infection, periarticular fracture, neuropathic arthropathy, reactive arthritis, gout or a pregnancy. In a trial analysis prior to the main study, it was observed that 5 of 27 patients showed unaffected high BTU values after realignment surgery for relevant medial zones due to meniscal chondrocalcinosis and osteochondral lesions. These patients were excluded for further analyses (Fig. 1).

Medial opening-wedge HTO was performed in every patient according to published standard techniques by experienced orthopaedic surgeons [19, 21, 26, 27, 33].
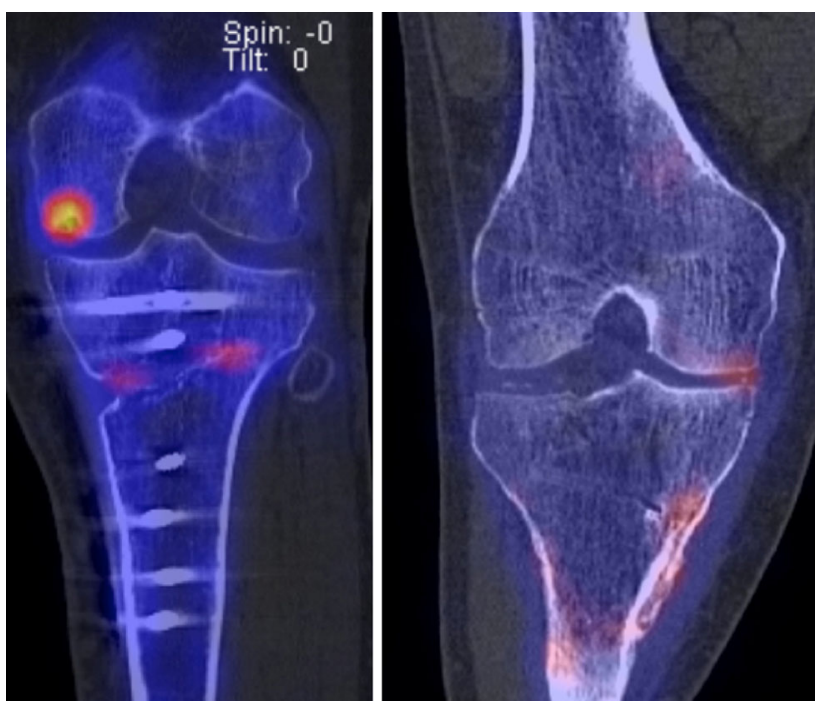

Fig. 1 Left SPECT/CT image of a 50-year-old male patient 12 months after HTO showing a consistently increased uptake on the medial femoral condyle due to OCL which is only partially affected by HTO. An increased tracer uptake can be seen in the osteotomy gap indicating advancing osseous consolidation. Right SPECT/CT image of a 50-year-old male patient 13 months after HTO showing medial meniscal chondrocalcinosis with increased tracer uptake 

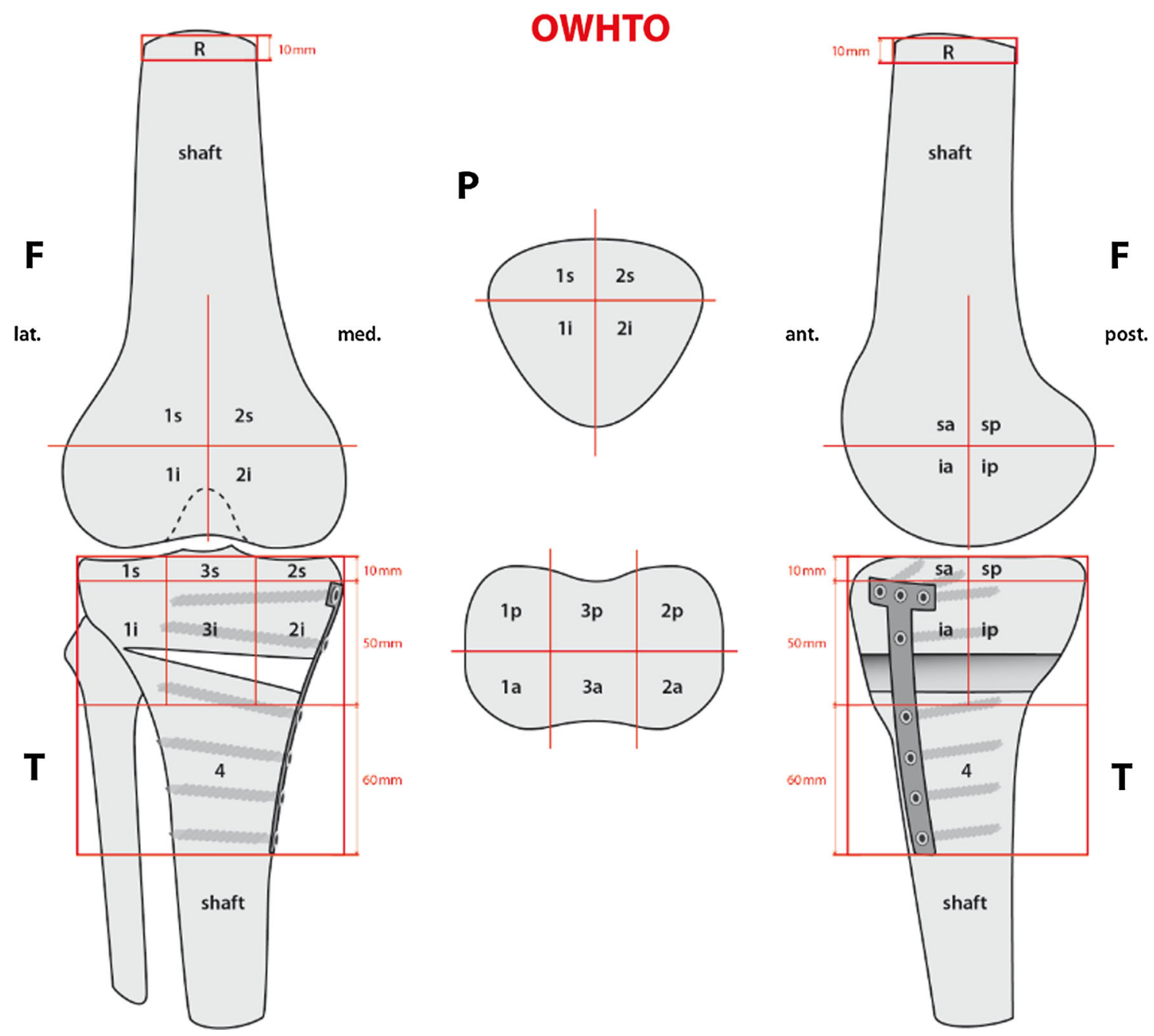

Fig. 2 The mapping scheme used for localization of areas of increased SPECT/CT tracer uptake values in knees after HTO $(F$ femur, $T$ tibia, $P$ patella, $R$ reference zone, 1 lateral, 2 medial, 3 tibial intercondylar area, 4 distal plate area, $s$ superior, $i$ inferior, $a$ anterior, $p$ posterior)

All procedures were performed in accordance with the ethical standards of the responsible committee and with the guidelines of the Helsinki Declaration of 1975 , as revised in 2008 .

Anterior-posterior (AP) and lateral weight-bearing radiographs as well as a $45^{\circ}$ posterior-anterior view and skyline views were routinely obtained before HTO, 4 months, 1 and 2 years postoperatively. Long leg weightbearing radiographs (pelvis to ankle) were performed preoperatively and 4 months postoperatively.

99mTc-HDP-SPECT/CT was performed preoperatively, 12 and/or 24 months after HTO using our previously published 4D-SPECT/CT protocol [29]. All SPECT/CTs were performed using a hybrid system (Symbia T16, Siemens, Erlangen, Germany). Planar scintigraphic images were taken in three phases, the perfusion phase (immediately after injection), the blood pool phase (2-5 min after injection) and the delayed metabolic phase (at least $2 \mathrm{~h}$ after injection). Two to three hours after injection of a commercial $500 \mathrm{MBq} 99$ m-Tc-HDP (Malinckrodt, Wollerau, Switzerland), a SPECT was performed with a matrix size of $128 \times 128$, an angle step of $32^{\circ}$ and a time per frame of $25 \mathrm{~s}$.

Bone tracer uptake was assessed in SPECT/CT images using a customized software (IntroSPECT, OrthoImagingSolutions Ltd., London, UK), which is able to quantify bone tracer uptake volumetrically in 3D and localize the areas of increased or decreased BTU to specific biomechanical relevant joint areas [16]. For localization of the BTU, a previously validated standardized localization scheme was used (Figs. 2, 3), which was specifically tailored for knees after HTO. In order to have comparable SPECT volumes, we used an identical localization scheme, which was adjusted to both native and operated knees. The scheme defines 9 femoral, 8 patellar and 13 tibial zones to accurately map the examined tracer uptake volume. The 


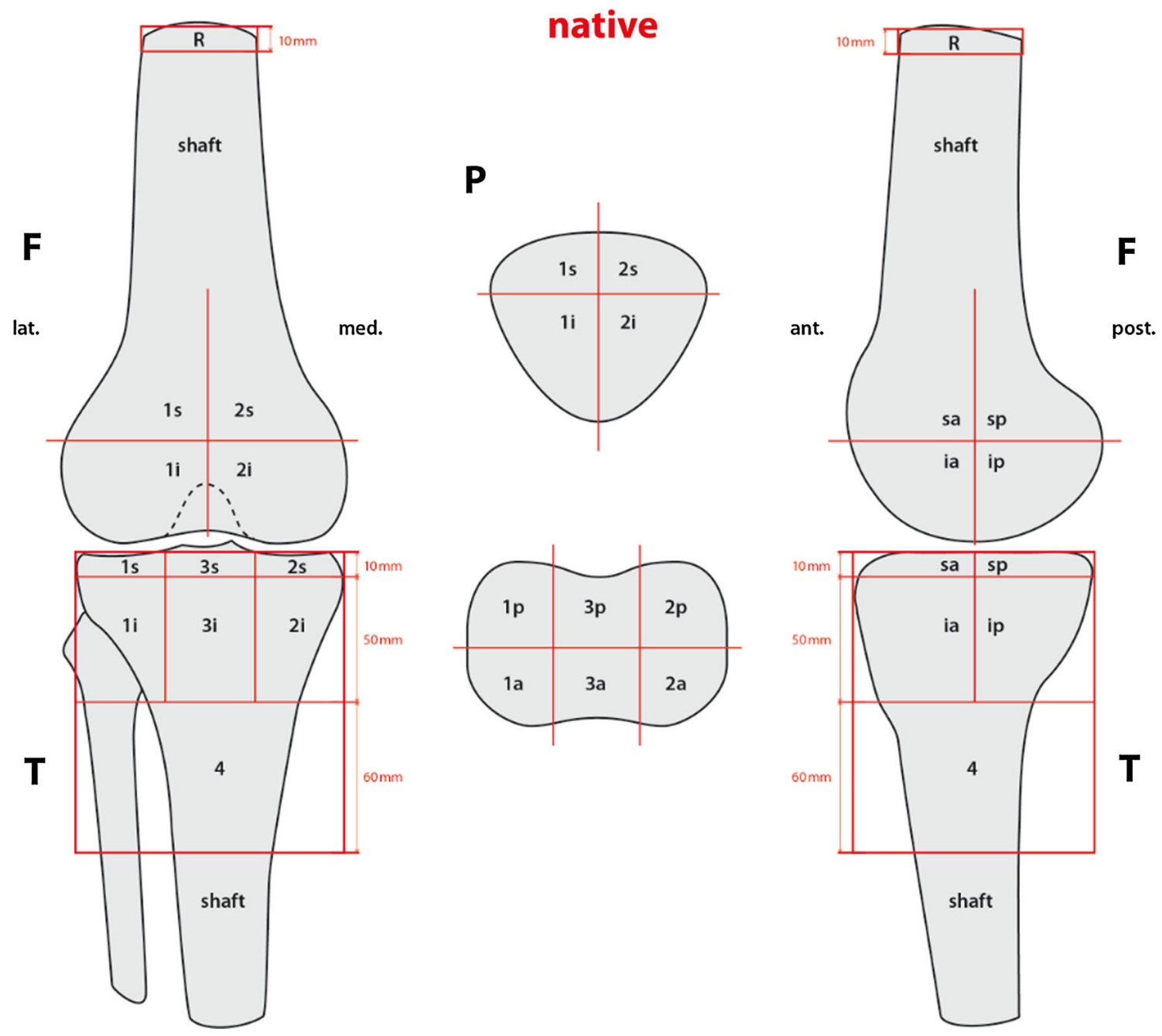

Fig. 3 The mapping scheme used for localization of areas of increased SPECT/CT tracer uptake values in native knees $(F$ femur, $T$ tibia, $P$ patella, $R$ reference zone, 1 lateral, 2 medial, 3 tibial intercondylar area, 4 distal plate area, $s$ superior, $i$ inferior, $a$ anterior, $p$ posterior)

femur $(\mathrm{F})$ is divided into nine zones that include one reference zone in the shaft $(\mathrm{R})$ and eight distal femoral zones. Each distal femoral zone is represented with a number (1lateral, 2-medial) and 2 small letters (a-anterior, p-posterior and i-inferior, s-superior). The tibia ( $\mathrm{T}$ ) is divided into 13 zones that include each six proximal and distal tibial regions and one shaft region. Each tibial zone is represented with a number (1-lateral plateau, 2-medial plateau, 3-tibial spine) and two small letters (a-anterior, p-posterior and i-inferior, s-superior). In the tibia, the thickness of the superior zones was defined as $10 \mathrm{~mm}$ to mimic the subchondral bone plate as close as possible. Inferior tibial zones include the osteotomy site and are defined by a thickness of $50 \mathrm{~mm}$. Zone 4 represents the distal part of the plate including screws. The patella (P) is divided into four zones (superomedial, superolateral, inferomedial and inferolateral).
Maximum values (mean \pm standard deviation, median and range) for each area of the localization scheme were recorded as well as normalized relative values for intensity of bone tracer uptake calculated. For normalization, the femoral shaft was used as reference region as previously published [16].

Pre- and postoperative mechanical alignment was measured in degrees on SPECT/CT using a previously validated custom-made specialized software (Orthoexpert v1.15, OrthoImagingSolutions Ltd., London, UK) [29]. The localization scheme showed near-perfect inter- and intra-OR (intra-class correlation coefficient (ICC) $>0.9$ ) for the measurement of tracer activity and localization in all anatomical regions. For measurements of mechanical alignment, there was a strong agreement between the two observers (an inter-OR of ICC $=0.99$ and an intra-OR of ICC $=0.98)$ [25]. 
Table 1 Mean, standard deviation, minimum and maximum of grading for normalized values of SPECT/CT tracer uptake for relevant areas of the localization scheme for the difference of preoperative and postoperative measurements, including Wilcoxon's signed-rank test for the differences

\begin{tabular}{|c|c|c|c|c|c|c|c|c|c|c|c|c|c|}
\hline$N=23$ & $1 \mathrm{saFe}$ & $2 \mathrm{saFe}$ & $1 \mathrm{spFe}$ & \multicolumn{2}{|c|}{$2 \mathrm{spFe}$} & liaFe & $2 \mathrm{iaFe}$ & 1ipFe & 2ipFe & 1sPat & $2 \mathrm{sPat}$ & 1iPat & 2iPat \\
\hline \multicolumn{14}{|c|}{ Difference pre- and postoperative measurements } \\
\hline Mean & -0.29 & -0.3 & -0.5 & \multicolumn{2}{|c|}{-0.45} & -0.35 & -1.42 & -0.28 & -1.81 & -0.78 & -0.5 & -0.55 & -0.41 \\
\hline Median & -0.32 & -0.28 & -0.3 & -0.42 & & -0.37 & -0.84 & -0.25 & -1.51 & -0.6 & -0.51 & -0.53 & -0.33 \\
\hline SD & 0.46 & 0.5 & 0.82 & \multicolumn{2}{|c|}{0.91} & 0.62 & 1.37 & 0.65 & 1.55 & 1.26 & 0.93 & 0.71 & 0.59 \\
\hline Min & -0.97 & -1.52 & -3.36 & -3.01 & -1 . & & -4.39 & -2.18 & -5.7 & -3.66 & -2.52 & -2.03 & -1.76 \\
\hline Max & 0.97 & 0.89 & 0.53 & 1.51 & & .06 & 0.65 & 0.94 & 0.12 & 2.19 & 1.54 & 0.87 & 0.84 \\
\hline Wilcox & test & & & & & & & & & & & & \\
\hline Z & -2.92 & -2.95 & -3.3 & -2.68 & -2 . & & -4 & -1.82 & -4.17 & -2.89 & -2.28 & -3.35 & -3.15 \\
\hline $\mathrm{p}$ & 0.0035 & 0.003 & 0.00 & 0.00 & & .0078 & 0.0001 & 0.068 & 0 & 0.0039 & 0.0225 & 0.0008 & 0.0016 \\
\hline$N=23$ & 1saTib & 3saTib & 2saTib & 1spTib & 3 spTib & 2 spTib & 1iaTib & 3iaTib & 2iaTib & 1ipTib & 3ipTib & 2ipTib & 4Tib \\
\hline Differen & e pre- and & l postoperat & tive measur & ements & & & & & & & & & \\
\hline Mean & -0.2 & -0.32 & -1.02 & 0.16 & 0.18 & -1.28 & 0.91 & 1.52 & 1.42 & 2.32 & 3.97 & 3.14 & 1.16 \\
\hline Median & -0.14 & -0.09 & -0.78 & -0.08 & -0.04 & -1.02 & 0.6 & 1.31 & 1.54 & 1.29 & 2.08 & 1.81 & 0.5 \\
\hline SD & 0.5 & 0.69 & 1.36 & 0.87 & 0.88 & 1.75 & 1.84 & 2.2 & 2.02 & 3.58 & 4.55 & 4.1 & 1.88 \\
\hline Min & -1.86 & -2.43 & -6.04 & -1.17 & -0.9 & -8.11 & -1.33 & -1.26 & -4.12 & -0.53 & 0.38 & -5.07 & -0.29 \\
\hline Max & 0.57 & 0.69 & 0.41 & 2.79 & 2.41 & 0.68 & 7.71 & 8.24 & 6.48 & 12.87 & 17.96 & 10.47 & 7.77 \\
\hline Wilcoxor & test & & & & & & & & & & & & \\
\hline Z & -1.56 & -2.28 & -3.76 & -0.18 & -0.26 & -3.98 & -2.59 & -2.98 & -3.2 & -3.67 & -4.2 & -3.41 & -3.44 \\
\hline $\mathrm{p}$ & 0.119 & 0.0225 & 0.0002 & 0.8552 & 0.7951 & 0.0001 & 0.0097 & 0.0029 & 0.001 & 0.0002 & 0 & 0.0007 & 0.0006 \\
\hline
\end{tabular}

Clinical outcome was assessed at 24 months after HTO using the German version of the WOMAC score [34].

The study was approved by the local ethical committee.

Statistical analysis

Data were analysed using SPSS 17.0 (SPSS, Chicago, IL). Means of two measurements of one observer were applied for statistical analysis.

A nonparametric Spearman's correlation was used to compute associations between the WOMAC score and the mechanical alignment with the tracer uptake in each relevant area. Paired $t$ test/Wilcoxon rank sum-test was used to compare specific areas for pre- and postoperative SPECT/ CT tracer uptake. For all analyses, $p<0.05$ was considered statistically significant.

\section{Results}

A significant decrease of BTU in the medial subchondral zones after HTO was found from preoperatively to 12 and 24 months follow-up $(p<0.01)$. BTU normalized in all asymptomatic patients within 24 months. The normalized grading of BTU in SPECT/CT for each anatomical area of the localization scheme is presented in Table 1 (values represent difference between preoperative and postoperative measurements).

A decrease of BTU was partly seen in the lateral compartments, but the decrease was significantly higher in the deloaded medial tibial and femoral joint compartment $(p<0.0001$, Fig. 4).

The achieved average valgus correction of the tibiofemoral angle by HTO was $5.9^{\circ} \pm 2.8^{\circ}$. There were no adverse events such as pseudoarthrosis, infection, loss of correction or skin necrosis. The mean WOMAC score pain (0-20) was 6.2 \pm 5.6, WOMAC stiffness (0-8) was $2.8 \pm 2.4$, and the WOMAC daily activities (0-68) was $17.4 \pm 16.4$. The mean total score (0-96) was $25.4 \pm 22.00$ after HTO (Fig. 5).

Less stiffness with regard to the WOMAC score correlated significantly with a higher decrease in SPECT/CT BTU $(p<0.05)$. Higher postoperative bone tracer uptake significantly correlated with more pain $(p<0.05)$. A Spearman correlation analysis revealed no statistical significant associations between SPECT/CT BTU and alignment correction by HTO.

\section{Discussion}

The findings of this study have led to a better understanding of the in vivo loading of the different joint 

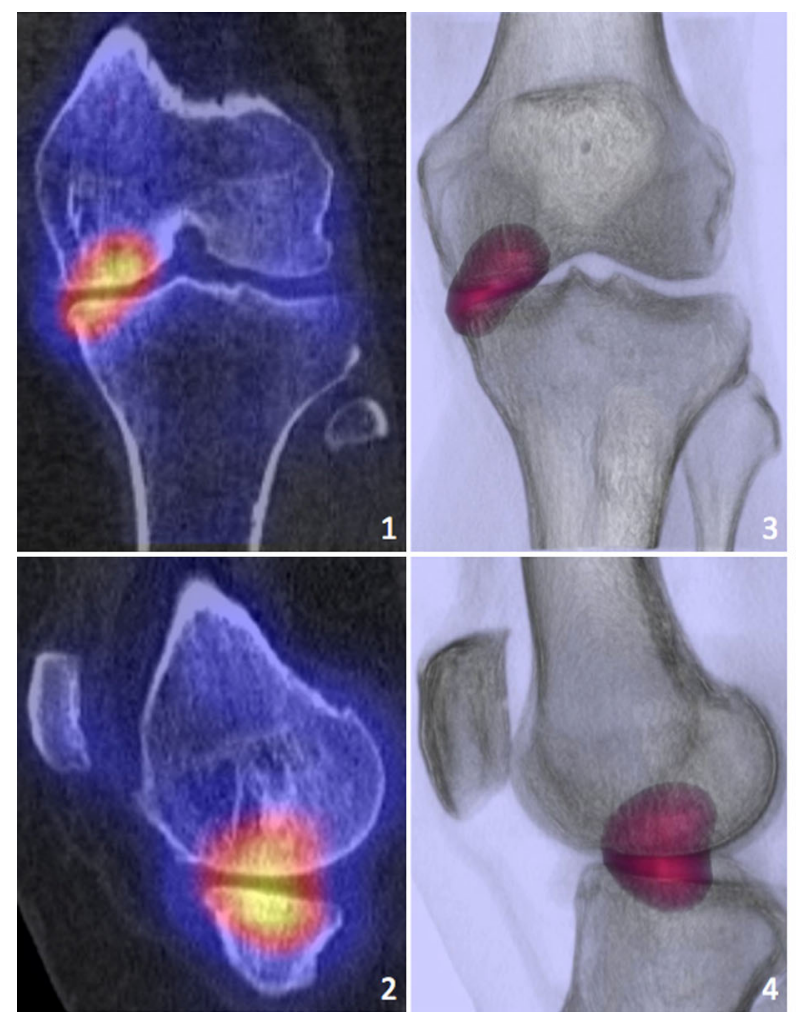

Fig. 4 Left SPECT/CT images $(\mathbf{1}, 2)$ and 3D radiolucent reconstructions $(3,4)$ of a 52 -year-old female patient before HTO showing medial overloading. Right SPECT/CT images $(5,6)$ and 3D

compartments before and after HTO. The most important findings and implications of this study were the following:

Firstly, before HTO surgery, all patients showed an increased BTU in the medial compartment visualizing the medial compartment overloading. A significant decrease of BTU, which reflects the in vivo loading of the bone, was noted in the medial subchondral compartment zones after HTO. This is the first time the deloading effect of HTO was shown by musculoskeletal imaging in an in vivo situation. The average correction of $5.5^{\circ}$ towards valgus led to a significant deloading effect in the medial compartment. In this study, the average postoperative mechanical axis was $2.8^{\circ}$ of valgus compared to a preoperative mechanical axis of $2.7^{\circ}$ of varus, and these findings are in accordance with McNamara et al., who recommended rather a slight correction than overcorrection with the inherent risk of disease progression in the lateral compartment [24]. In that study, it was also concluded that a significant reduction of the adduction moment is achievable without large overcorrections of the mechanical axis [24].

Despite shifting the weight-bearing line to the lateral compartment, no increased postoperative BTU was found in the lateral tibial or femoral zones. It can be concluded that the performed average correction in this study did not cause overloading within the 24-month follow-up period.
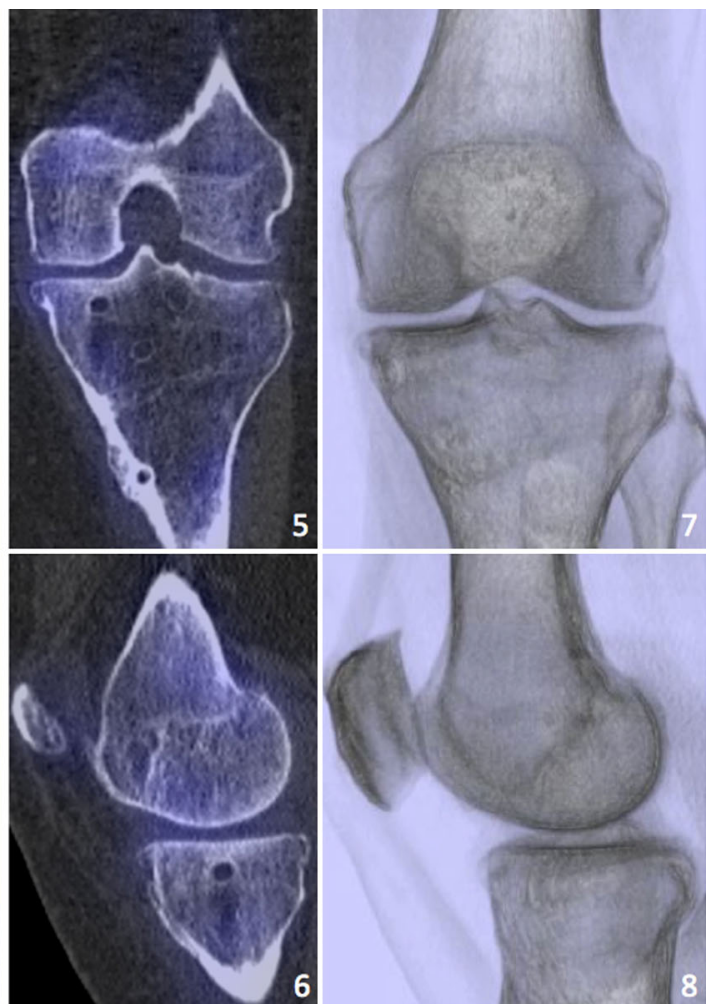

reconstructions $(\mathbf{7}, \mathbf{8}) 16$ months postoperatively reveal an unloading effect of the medial joint compartment and a consolidation of the osteotomy gap

This is important as Sharma et al. [31] showed that valgus alignment (mechanical axis $\geq 2^{\circ}$ valgus) can increase the risk of OA progression in the affected compartment. Similar findings were shown by Hernigou et al. [11], who reported a series of progressive lateral compartment degeneration in overcorrected knees over the follow-up time.

However, Ziegler et al. [38] found no significant macroscopic and microscopic structural changes in the sheep cartilage of the lateral tibial plateau and femoral condyle after 6 months in vivo. They further emphasized the fragile equilibration of load dissipation, which could only work with an intact meniscus. In patients undergoing HTO, not rarely the medial and lateral meniscus do show some tears or degeneration [38].

In this study, SPECT/CT has proven to be beneficial for follow-up of patients before and after HTO. Due to its unique characteristics, SPECT/CT offers the chance to gain information on the loading of the different compartments in the knee [12-15]. SPECT/CT is able to show the loading history of the knee, and thus, overloading can be assessed at an early stage. SPECT/CT could be further used to answer the open question where to shift the weight-bearing line for optimal deloading in each patient. Generally, the surgeon aims for a point $62 \%$ laterally on the tibial plateau 

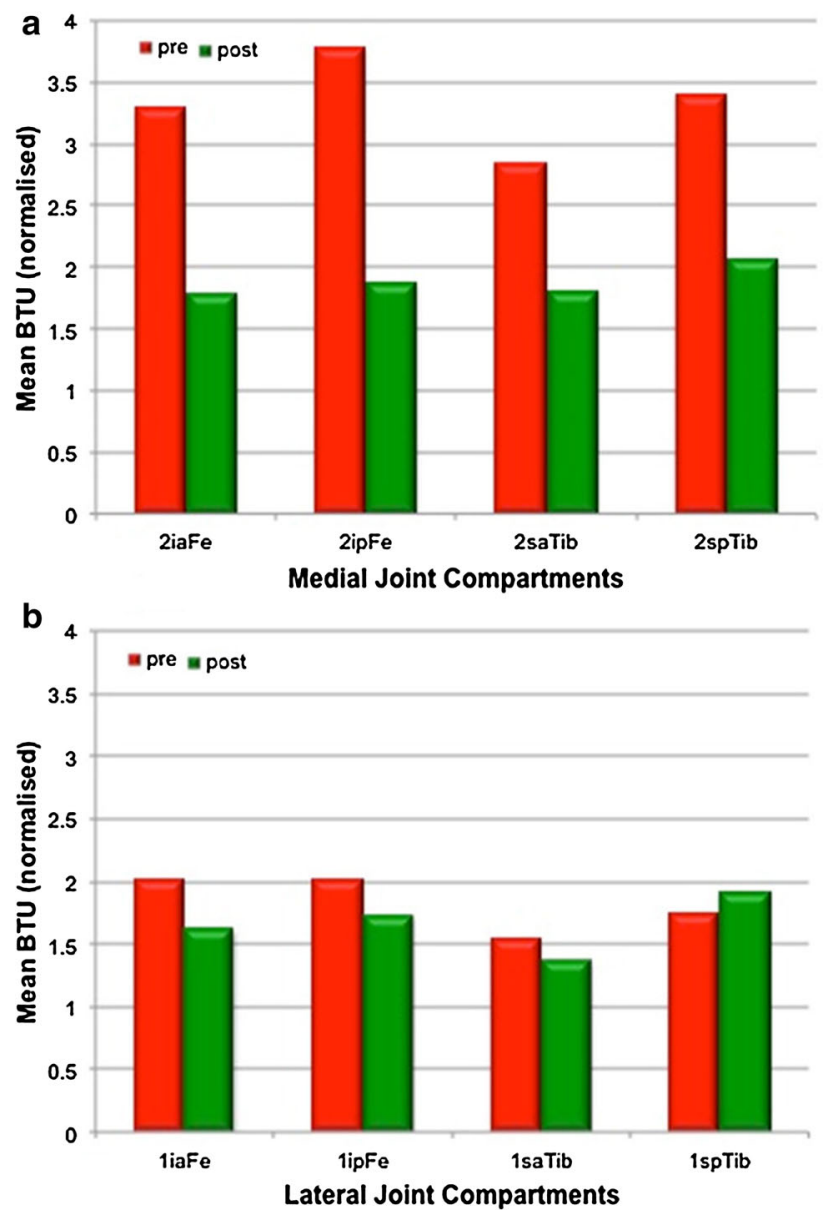

Fig. 5 a, b Normalized pre- and postoperative BTU intensity in the medial (2) and lateral (1) subchondral tibial and femoral joint compartments showing a significant decrease of BTU in the medial joint compartments and a consistent BTU in lateral joint compartments. $F e$ femur, Tib tibia, $i$ inferior, $s$ superior, $a$ anterior, $p$ posterior

diameter (Fujisawa point), but it is still unclear whether this aim is correct in every case. In some patients, it could lead to under- or overcorrection. The optimal individualized correction for each patient remains controversial. This statement is in agreement with Amis [1], who emphasized the fact that walking is a dynamic activity. In addition, patients individually adapt their gait and muscle activation to their experienced problems leading to change of loading patterns. Making things even more complicated, varus alignment and varus thrust is not only a problem for the medially compressed bony compartment but also for the laterally stretched soft tissue structures [35]. Striving for a more individualized guideline for limb alignment correction, Marti et al. [23] suggested to individually tailor the degree of correction of leg alignment with regard to the degree of cartilage loss in the affected knee compartment.

Secondly, the decrease of BTU in other compartments than the deloaded medial ones can be explained by general normalization of bone metabolism and inflammation after HTO. The significant increase of bone tracer uptake in zones directly adjacent to the plate or screws or within the osteotomy zone reflects the consolidation of the osteotomy gap and the bony remodelling. Interestingly, bone tracer uptake decreased but persisted over the 24-month followup. It is commonly assumed that the osteotomy gap is consolidated within one year after surgery and removal of the osteosynthesis plate is mostly performed within $8-12$ months postoperatively, but the healing is not complete at this time [26].

In addition, it was shown that pathologies such as meniscal chondrocalcinosis and osteochondral lesions result in increased bone tracer uptake, even after deloading. As reported by Konala et al. [20], SPECT/CT allows the surgeon to judge osteochondral lesions (OCL). Thus, it was observed that three patients with isolated areas of massively increased tracer uptake, which were intraoperatively confirmed as osteochondral lesions, showed no significant decrease of uptake after HTO, implying that the osteochondral lesion remains "hot" even after HTO. In contrast, the clinical symptoms of these patients resolved. It seems that the underlying pathology determines the appearance in SPECT/CT. However, this fact needs to be further addressed in larger scaled clinical studies.

In this study, two patients with signs of meniscal chondrocalcinosis in plain radiographs showed increased bone tracer uptake in these zones, assuming that the utilized $99 \mathrm{mTc}-\mathrm{HDP}$ tracer substance is linking to calcium pyrophosphate which is amassed in the calcinotic meniscus [7]. Since meniscal chondrocalcinosis is known to be an important factor in the pathogenesis of OA, this observation warrants further investigation in a separate study $[7,10]$.

Thirdly, a significant correlation of stiffness and pain with the higher decrease in SPECT/CT BTU was found. Higher postoperative BTU significantly correlated with more pain. There was, however, no statistical significant association between BTU intensity and alignment correction by HTO, which is rather a result of the small sample size than reflecting a real finding.

However, W-Dahl et al. also failed to show a significant association of knee alignment and knee pain preoperatively and at 12 months postoperatively in patients undergoing HTO for varus malalignment [36]. They explained their findings by the conclusion that knee alignment and pain are different entities. It seems that for successful joint-preserving therapy, a number of different structures and factors such as integrity of the meniscus, cartilage and ligaments as well as the alignment have to be taken into consideration [2].

In contrast, it was reported by Briem et al. [3] that over- or undercorrected patients had significantly less improvement in 
their KOS-ADLS scores. The finding was attributed to higher medial and lateral co-contractions and adduction moments 1 year after surgery.

With regard to the results of our study, there are certainly some limitations that have to be considered when interpreting our data. The indication for HTO might have been affected by the preferences of the different surgeons. This could have led to a selection bias. However, the patients included represent a typical series found in orthopaedic knee clinics [30].

As the case number is rather small, significant associations between tracer uptake and mechanical alignment and/ or clinical outcome might not show significant correlations here, but could become significant with a larger sample size.

\section{Conclusion}

In patients with medial compartment, overloading due to varus malalignment HTO led to a significant decrease in bone tracer uptake in the medial joint compartments and normalization in asymptomatic patients after 24 months. SPECT/CT tracer uptake patterns and intensity distribution in these patients from pre- to 12 and 24 months postoperatively correlated significantly with pain and stiffness. Hence, SPECT/CT could be used for assessment of adequate correction and healing after HTO.

Acknowledgments We greatly thank the Deutsche Arthrose Hilfe e.V., Germany, for financial support of the study and Jürg Schmutz for his illustrative work explaining the algorithm.

\section{References}

1. Amis AA (2013) Biomechanics of high tibial osteotomy. Knee Surg Sports Traumatol Arthrosc 21:197-205

2. Arnold MP, Hirschmann MT, Verdonk PC (2012) See the whole picture: knee preserving therapy needs more than surface repair. Knee Surg Sports Traumatol Arthrosc 20:195-196

3. Briem K, Ramsey DK, Newcomb W, Rudolph KS, SnyderMackler L (2007) Effects of the amount of valgus correction for medial compartment knee osteoarthritis on clinical outcome, knee kinetics and muscle co-contraction after opening wedge high tibial osteotomy. J Orthop Res 25:311-318

4. Brouwer GM, van Tol AW, Bergink AP, Belo JN, Bernsen RM, Reijman M, Pols HA, Bierma-Zeinstra SM (2007) Association between valgus and varus alignment and the development and progression of radiographic osteoarthritis of the knee. Arthritis Rheum 56:1204-1211

5. Coventry MB (1965) Osteotomy of the upper portion of the tibia for degenerative arthritis of the knee. A preliminary report. J Bone Joint Surg Am 47:984-990

6. Crema MD, Roemer FW, Felson DT, Englund M, Wang K, Jarraya M, Nevitt MC, Marra MD, Torner JC, Lewis CE, Guermazi A (2012) Factors associated with meniscal extrusion in knees with or at risk for osteoarthritis: the Multicenter Osteoarthritis study. Radiology 264:494-503

7. Derfus BA, Kurian JB, Butler JJ, Daft LJ, Carrera GF, Ryan LM, Rosenthal AK (2002) The high prevalence of pathologic calcium crystals in pre-operative knees. J Rheumatol 29:570-574

8. Dugdale TW, Noyes FR, Styer D (1992) Preoperative planning for high tibial osteotomy. The effect of lateral tibiofemoral separation and tibiofemoral length. Clin Orthop Relat Res 274:248-264

9. Eckstein F, Hudelmaier M, Cahue S, Marshall M, Sharma L (2009) Medial-to-lateral ratio of tibiofemoral subchondral bone area is adapted to alignment and mechanical load. Calcif Tissue Int 84:186-194

10. Fuerst M, Lammers L, Schafer F, Niggemeyer O, Steinhagen J, Lohmann CH, Ruther W (2010) Investigation of calcium crystals in OA knees. Rheumatol Int 30:623-631

11. Hernigou P, Medevielle D, Debeyre J, Goutallier D (1987) Proximal tibial osteotomy for osteoarthritis with varus deformity. A ten to thirteen-year follow-up study. J Bone Joint Surg Am 69:332-354

12. Hirschmann MT, Adler T, Rasch H, Hugli RW, Friederich NF, Arnold MP (2010) Painful knee joint after ACL reconstruction using biodegradable interference screws-SPECT/CT a valuable diagnostic tool? A case report. Sports Med Arthrosc Rehabil Ther Technol 2:24

13. Hirschmann MT, Davda K, Rasch H, Arnold MP, Friederich NF (2011) Clinical value of combined single photon emission computerized tomography and conventional computer tomography $(\mathrm{SPECT} / \mathrm{CT})$ in sports medicine. Sports Med Arthrosc 19:174-181

14. Hirschmann MT, Schmid R, Dhawan R, Skarvan J, Rasch H, Friederich NF, Emery R (2011) Combined single photon emission computerized tomography and conventional computerized tomography: clinical value for the shoulder surgeons? Int J Shoulder Surg 5:72-76

15. Hirschmann MT, Schon S, Afifi FK, Amsler F, Rasch H, Friederich NF, Arnold MP (2013) Assessment of loading history of compartments in the knee using bone SPECT/CT: a study combining alignment and 99mTc-HDP tracer uptake/distribution patterns. J Orthop Res 31:268-274

16. Hirschmann MT, Wagner CR, Rasch H, Henckel J (2012) Standardized volumetric 3D-analysis of SPECT/CT imaging in orthopaedics: overcoming the limitations of qualitative 2D analysis. BMC Med Imaging 12:5

17. Jackson JP, Waugh W (1961) Tibial osteotomy for osteoarthritis of the knee. J Bone Joint Surg Br 43:746-751

18. Johnson F, Leitl S, Waugh W (1980) The distribution of load across the knee. A comparison of static and dynamic measurements. J Bone Joint Surg Br 62:346-349

19. Kolb W, Guhlmann H, Windisch C, Kolb K, Koller H, Grutzner P (2009) Opening-wedge high tibial osteotomy with a locked lowprofile plate. J Bone Joint Surg Am 91:2581-2588

20. Konala P, Iranpour F, Kerner A, Rasch H, Friederich NF, Hirschmann MT (2010) Clinical benefit of SPECT/CT for follow-up of surgical treatment of osteochondritis dissecans. Ann Nucl Med 24:621-624

21. Lobenhoffer P, Agneskirchner JD (2003) Improvements in surgical technique of valgus high tibial osteotomy. Knee Surg Sports Traumatol Arthrosc 11:132-138

22. Lustig S, Scholes CJ, Costa AJ, Coolican MJ, Parker DA (2013) Different changes in slope between the medial and lateral tibial plateau after open-wedge high tibial osteotomy. Knee Surg Sports Traumatol Arthrosc 21:32-38

23. Marti CB, Gautier E, Wachtl SW, Jakob RP (2004) Accuracy of frontal and sagittal plane correction in open-wedge high tibial osteotomy. Arthroscopy 20:366-372 
24. McNamara I, Birmingham TB, Fowler PJ, Giffin JR (2013) High tibial osteotomy: evolution of research and clinical applications-a Canadian experience. Knee Surg Sports Traumatol Arthrosc 21:23-31

25. Mucha A, Dordevic M, Testa EA, Rasch H, Hirschmann MT (2013) Assessment of the loading history of patients after high tibial osteotomy using SPECT/CT - a new diagnostic tool and algorithm. J Orthop Surg Res 8:46

26. Niemeyer P, Koestler W, Kaehny C, Kreuz PC, Brooks CJ, Strohm PC, Helwig P, Suedkamp NP (2008) Two-year results of open-wedge high tibial osteotomy with fixation by medial plate fixator for medial compartment arthritis with varus malalignment of the knee. Arthroscopy 24:796-804

27. Niemeyer P, Schmal H, Hauschild O, von Heyden J, Sudkamp NP, Kostler W (2010) Open-wedge osteotomy using an internal plate fixator in patients with medial-compartment gonarthritis and varus malalignment: 3-year results with regard to preoperative arthroscopic and radiographic findings. Arthroscopy 26:16071616

28. Pape D, Seil R, Adam F, Rupp S, Kohn D, Lobenhoffer P (2004) Imaging and preoperative planning of osteotomy of tibial head osteotomy. Orthopade 33:122-134

29. Rasch H, Falkowski AL, Forrer F, Henckel J, Hirschmann MT (2013) 4D-SPECT/CT in orthopaedics: a new method of combined quantitative volumetric 3D analysis of SPECT/CT tracer uptake and component position measurements in patients after total knee arthroplasty. Skeletal Radiol 42:1215-1223

30. Schaefer TK, Majewski M, Hirschmann MT, Friederich NF (2008) Comparison of sagittal and frontal plane alignment after open- and closed-wedge osteotomy: a matched-pair analysis. J Int Med Res 36:1085-1093
31. Sharma L, Song J, Dunlop D, Felson D, Lewis CE, Segal N, Torner J, Cooke TD, Hietpas J, Lynch J, Nevitt M (2010) Varus and valgus alignment and incident and progressive knee osteoarthritis. Ann Rheum Dis 69:1940-1945

32. Sharma L, Song J, Felson DT, Cahue S, Shamiyeh E, Dunlop DD (2001) The role of knee alignment in disease progression and functional decline in knee osteoarthritis. JAMA 286:188-195

33. Staubli AE, De Simoni C, Babst R, Lobenhoffer P (2003) TomoFix: a new LCP-concept for open wedge osteotomy of the medial proximal tibia-early results in 92 cases. Injury 34(Suppl 2):B55-B62

34. Stucki G, Meier D, Stucki S, Michel BA, Tyndall AG, Dick W, Theiler R (1996) Evaluation of a German version of WOMAC (Western Ontario and McMaster Universities) Arthrosis Index. Z Rheumatol 55:40-49

35. van de Pol GJ, Arnold MP, Verdonschot N, van Kampen A (2009) Varus alignment leads to increased forces in the anterior cruciate ligament. Am J Sports Med 37:481-487

36. W-Dahl A, Toksvig-Larsen S, Roos EM (2009) Association between knee alignment and knee pain in patients surgically treated for medial knee osteoarthritis by high tibial osteotomy. A one year follow-up study. BMC Musculoskelet Disord 10:154

37. Wenham CY, Conaghan PG (2009) Imaging the painful osteoarthritic knee joint: what have we learned? Nat Clin Pract Rheumatol 5:149-158

38. Ziegler R, Goebel L, Cucchiarini M, Pape D, Madry H (2013) Effect of open wedge high tibial osteotomy on the lateral tibiofemoral compartment in sheep. Part II: standard and overcorrection do not cause articular cartilage degeneration. Knee Surg Sports Traumatol Arthrosc. doi:10.1007/s00167-013-2410-6 